\title{
A Risk-Aware Application Scheduling Model in Cloud Computing Scenarios
}

\author{
Ala Arman \\ DTI - Università degli Studi di Milano, Crema, 26013, Italia \\ E-mail: ala.arman@unimi.it
}

\begin{abstract}
Cloud users usually have different preferences over their applications that outsource to the cloud, based on the financial profit of each application's execution. Moreover, various types of virtual machines are offered by a cloud service provider with distinct characteristics, such as rental prices, availability levels, each with a different probability of occurrence and a penalty, which is paid to the user in case the virtual machine is not available. Therefore, the problem of application scheduling in cloud computing environments, considering the risk of financial loss of application-to-VM assignment becomes a challenging issue. In this paper, we propose a riskaware scheduling model, using risk analysis to allocate the applications to the virtual machines, so that, the expected total pay-off of an application is maximized, by taking into account of the priority of applications. A running example is used through the paper to better illustrate the model and its application to improve the efficiency of resource as signment in cloud computing scenarios.
\end{abstract}

Index Terms-Application scheduling, cloud computing, expected monetary value, risk analysis.

\section{INTRODUCTION}

Cloud computing [1] is a potent Internet-based service, which offers a new technique to provide huge amount of shared resources offered by several cloud service providers (CSPs). There are two main advantages for adopting a cloud solution [2], which have been discussed in the literature. First, the end user does not need to be involved in the configuration and maintenance of cloud services provided by the CSP. Second, thanks to "pay-as-you-go" pricing model, the end-user only pays for the resources that requests and uses. Therefore, she can evade unnecessary costs [3], specially the high in itial cost of setting up the application deployment environment. Additionally, there are other advantages of cloud computing discussed in the literature [4]-[6], like high elasticity and availability, reliability, multi-tenancy, on demand self-service, broad network access, etc.

Therefore, an increasing number of users tend to outsource their existing applications to the cloud environments in order to benefit from their significant features. However, application scheduling, that is, assign the available resources on a cloud environment to an application, has become a prominent problem in cloud scenarios because it directly affects the application's performance. In worse cases, poor application scheduling will result in the violation of quality of service (QoS) or even application failure.

Also, the CSPs provide several types of VMs to the users, in order to run their applications on the cloud, considering their heterogeneous requirements. For example, Amazon Elastic Compute Cloud (Amazon EC2) [7], is a web service that provides different types of VMs to the users. Each type of VM, which is offered by Amazon $\mathrm{EC} 2$, has different characteristics in terms of hourly rental price, available resource dimensions, such as CPU, RAM, disk space, network capacity, etc., to offer more flexibility for choosing appropriate type of VM(s) to be assigned to the user's applications, considering various requirements of each application. Furthermore, nowadays, the modern CSPs provide different levels of availability for the VMs, each with a service credit, in order to improve the management of service level agreement (SLA). Service credit is a percentage of (monthly in case of A mzaon EC2) rental charge of a $\mathrm{VM}$, which is paid to the user as a penalty, if the VM is not available. For example, currently, Amazon EC2 considers monthly uptime percentage $M U P$ of a VM to define its availability levels. Table presents different $M U P$ levels, as well as their related service credits [8] for the VMs, provided by Amazon EC2. For instance, if $M U P<99.0$, thirty percent of total monthly charge for renting the VM is paid to the user as a penalty and soon.

The cloud users usually have sensitive applications, so that, assigning them to the VMs inappropriately without considering the expected pay-off of each application's execution, could lead to huge financial loss of the user and decreasing her satisfaction by the offered cloud service significantly. As a result, the problem of assigning a set of applications with different financial execution profit, to a set of VMs, each with a rental price and several availability levels where each availability level is associated with a distinct probability of occurrence and a service cred it, so that, the expected pay-off for each application is maximized, considering the priority of applications, becomes a challenging is sue.

A naive approach to choose the most suitable VM for each application would consist in assigning an application with higher priority from the user's view to a cheaper VM.

However, such an approach would risk to ignore the several availability levels of VMs, each with a probability of occurrence and different service credit. To address this 
issue, in this paper, we propose a risk-aware application scheduling model aimed at decreasing the financial risk of application-to-VM assignment, considering the priority of applications defined by the user. To this purpose, our solution first produces, for each VM, a penalty which is paid to the user in case of SLA violation; Then it calculates the expected benefit of each application if it is assigned to each VM in $V$, considering the priority of applications; finally, it calculates the expected pay-off of each application, adopting a risk analysis technique (expected monetary value [9]), if it is assigned to each VM. Finally, we assign each application to a VM that the application has the highest expected pay-off with.

Table 1. An example for deferent levels for MUP and their service credits

\begin{tabular}{|c|c|}
\hline MUP level & $\begin{array}{c}\text { Service } \\
\text { Credit }\end{array}$ \\
\hline $\begin{array}{c}\text { Less than 99.95\% but equal to or greater } \\
\text { than } 99.0 \%\end{array}$ & $10 \%$ \\
\hline Less than $99.0 \%$ & $30 \%$ \\
\hline
\end{tabular}

The rest of this paper is as follows. Section II provides some related works that have been reported in this field. Section III briefly discusses about the research background of this paper, including cloud computing, virtualization technology, resource allocation in cloud environments, and risk analysis. Section IV presents our problem. Section V illustrates our solution. Finally, Section IV concludes the paper and discusses about future work.

\section{RELATED WORKS}

Previous works related to our proposal devoted to the assignment of a set of applications or tasks to a set of available VMs in a cloud domain, e.g., [10]-[16], that discuss how to assign applications or tasks, considering their different requirements as well as the characteristics of VMs. Most of these approaches fall short of considering the priority of applications, defined by the user. For example, the authors in [14] provide a scheduling strategy, considering multiple SLA parameters, for deploying applications in cloud environments, in order to optimize the performance of applications as well as reducing the possibilities of SLA violations. However, the authors did not consider the priority of applications, defined by the user in their scheduling approach. In addition, [16] offers a SLA-based resource provisioning solution in order to maximize the resource utilization and the profit of provider. The authors define multiple penalty types including a fixed penalty, a proportional penalty, and a delaydependent penalty in SLA. A penalty will incur if the number of SLA violations exceeds a predefined threshold. We see that their solution shares with us the view of considering penalty in SLA for assigning the applications to the VMs. However, contrary to our approach presented in this paper, an application with lower penalty rates has more priority from the CSP's view to be assigned to a
VM. Therefore, again here, the priority of applications is not based on the user's preferences. Moreover, few researches, e.g., [17], [18], have addressed the issues of application or task scheduling in cloud computing scenarios, while a user has different preferences over her applications or tasks. For instance, the authors in [17] propose an algorithm for dynamic allocation of VMs to a set of prioritized tasks based on multiple SLA parameters, such as me mory, network bandwidth, and requested CPU time by applying a preemption mechanis $\mathrm{m}$ for executing tas $\mathrm{ks}$ with higher priority. While our proposal and [17] share the idea of applying the priority of applications or tasks to schedule them, [17] does not take into account rental price and different availability levels of VMs, each with a probability of occurrence, and a service credit to schedule the tasks, which is one of the main contributions of this paper. Our approach strives to optimize the application scheduling in cloud computing scenarios by maximizing the expected total pay-off of each application-to-VM assignment, considering the priority of applications, which is according to the financial execution profit of each application.

\section{RESEARCH BACKGROUND}

This section briefly discusses some fundamental concepts of cloud computing and virtualization. Also, it describes the issues of resource allocation in cloud environments and the concept of expected monetary value (EMV) in quantitative risk analysis.

\section{A. Cloud Computing}

Cloud computing has become one of the most hottest and controversial topics in academic and industrial environments. It relies [19] on the practice of moving computing to the Internet. Intuitively, cloud users [20] outsource their data and applications to the cloud and access them remotely in a simple and pervasive way. In cloud computing environments, everything is offered as a service, that is, XaaS, e.g., SaaS (Software as a Service), PaaS (Platform as a Service), and IaaS (Infrastructure as a Service), which are three main service delivery models defined in cloud scenarios. They form a layered system structure for cloud computing, as presented in Fig.1. The most bottom layer (IaaS), which mostly is used by IT and network architects, is composed of physical and virtualized co mputing, storage, network resources, etc. The examples of IaaS providers are A mazon EC2, VMware [21], etc. The middle layer (PaaS), which is mostly used by application developers and testers, refers to offering platform layer resources, such as operating system support, software development frameworks, etc. The examples of PaaS providers include Google App Engine [22], Amazon SimpleDB [23], etc. The top layer (SaaS), which is used mostly by end-users, provides several types of ondemand cloud-based applications over the Internet. The examples of SaaS providers are Salesforce.com [24], Rackspace [25], etc. 


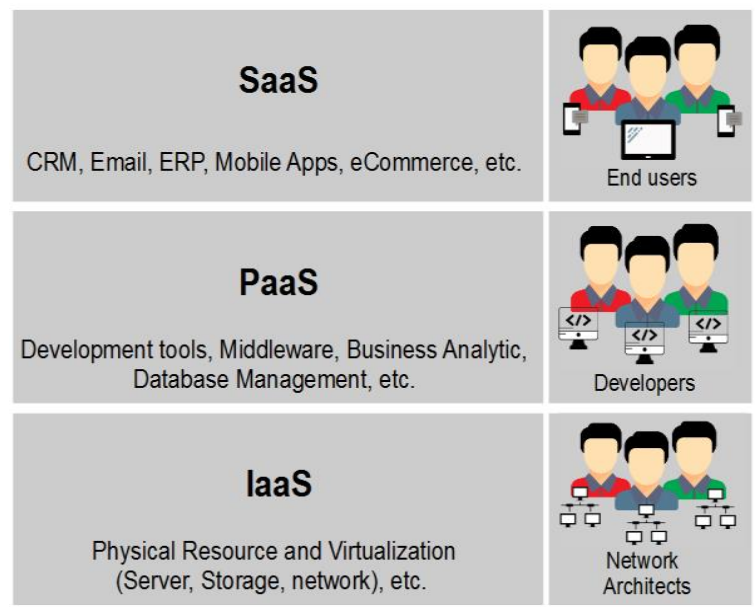

Fig.1. Cloud service delivery models

In cloud computing scenarios, since the demands of cloud user vary significantly during time, it is not poss ible to meet all of her requirements by the service(s) [26], which are provided by the CSPs. Also, it is essential that cloud users have guarantees from the CSPs [27], on service delivery. Therefore, in order to consider these challenges and requirements, a contract which is called service level agreement (SLA) is signed between a cloud user and a CSP, through a negotiation process. Two main aspects must be considered in a SLA: 1) quality of service $(Q o S)$ [28] require ments, that is, the measurable ability of a CSP to offer network and computation services, such that, the user's expectations from the offered service(s) are fulfilled, such as bandwidth availability, response time, CPU utilization, etc., 2) penalties, if QoS requirements are not met [29] by a provider. For example, as we discussed already in Section 0, Amazon EC2, provides QoS guaranties in multiple availability levels, with respect to the monthly uptime percentage $(M U P)$ of a VM. Also, it describes penalties in terms of service credits, which is a percentage of monthly rental charge of a VM.

\section{B. Virtualization}

Virtualization technology [30][31] is one of the most important key features of cloud computing, which refers to presenting the illusion of running many smaller VMs on a physical machine, each hosting a separate operating system instance. Simply put, each physical machine can run multiple VMs and each VM can be used by a different user, which is considered as one of the most crucial benefits of virtualization. Moreover, it is possible to utilize and assign different partitions of resources on the same physical machine to a VM. Also, since the resource requirements of a user change quickly due to the mobility of users, reallocation of resources is easier using virtualization [32] because virtual devices are software-based and offer a uniform interface through standard abstractions. In addition, leveraging virtualization technology, it is possible to encapsulate workloads and transfer them to idle or unused systems, which causes avoiding or delaying purchasing additional servers by consolidating existing systems. Fig.2, depicts a high level presentation of virtualization concept.

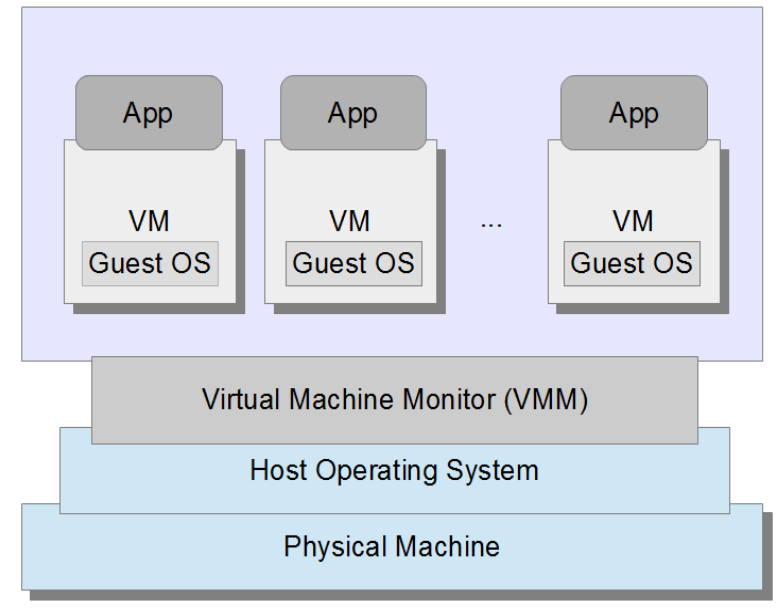

Fig.2. A general schema of virtualization

As can be seen, each VM uses an operating system, that is, the guest OS, which could be different from another VM on the same physical machine. Also, the virtual machine monitor (VMM) is a software layer, which mediates the interactions between a VM and the monitored host that the VM is running on. In other words, VMM masks [33] the complexities of physical machine from the guest execution environment.

\section{Resource Allocation In Cloud Enviroments}

Thanks to significant features of virtualization technology, which was partially discussed in Section 0 , the cloud users are able to run several applications, each with different characteristics and requirements on a set of VMs. However, application or task scheduling in a virtualized dynamic environment like cloud is not an easy task. In fact, due to the presence of heterogeneous types of resources in a cloud infrastructure as well as different tasks or applications, with various characteristics and requirements, the allocation of resources to tasks or applications in cloud computing environments is considered as a NPhard [34][35] (non-deterministic polynomial-time hard) problem. Therefore, it is so difficult to find an optimal solution in a polynomial time. The issue of resource allocation in cloud environments have been discussed in the literature, considering its several aspects such as security [36], resource consumption efficiency [15][37], etc. but the majority of previous studies have the limitation of not taking into account of the financial profit of a cloud user by running her tasks or applications on a cloud environment. A cloud user expects to maximize her financial profit, while migrating her applications to a cloud environment. Also, she usually has different preferences over her applications, according to the financial execution profit of each application, that is, an application with higher financial execution profit has higher priority from her view. The scenario gets more complex when in a virtualized data center, different VMs with various rental prices are provided by a CSP, with different availability levels, each with a probability of occurrence and a service credit. As a result, in such context, with high level of uncertainty, the application-to-VM assignment becomes a challenging issue. 
In this work, we resolve this is sue by using risk analysis techniques. In the next section, in order to be clearer, we will present some basic concepts in quantitative risk analysis, which is one of the main techniques to deal with risk and uncertainty. Also, we will discuss briefly about the expected monetary value (EMV) concept, which we will use later in this paper.

\section{Risk Analysis}

Risk [38] is an indicator of what could happen to assets of an organization if they are not properly protected. Risk analysis refers to a systematic review for estimating the magnitude of risks, which an organization is exposed. The main reason to perform risk analysis is [39] to support decision making in order to find the right balance between different concerns, such as cost, safety, etc. One of the most important applications of risk analysis is decision making under risk [15], which occurs when a decision maker is uncertain about the occurrence of a state of nature (event), but the probability of each state of nature is known. One of the most recommended quantitative tools and techniques for decision making under risk is called expected monetary value (EMV), which calculates the average outcome when the future includes scenarios that may or may not happen [40]. EMV for a course of action $j$, is the pay-off $X_{k j}$ for each combination of event $k$ multiplied by, the probability $\operatorname{Pr}_{k}$ of occurrence of event $k$, summed over all events. It is formally defined as follows:

$$
E M V_{j}=\sum_{k=1}^{d} \operatorname{Pr}_{k} X_{k j}
$$

The advantage of using EMV is considering uncertainty, by taking into account of a probability for each event $k$. Also, the problem becomes simpler by reducing the information about a course of action $j$ to a scalar value $E M V_{j}$.

\section{PROBLEM DEFINITION}

We consider a reference scenario like the one presented Fig.3., which is characterized by a user wishing to migrate to the cloud a set of $n$ applications $A=\left\{a_{1}, \ldots, a_{n}\right\}$. The user defines for each application $a_{i}$ a priority according to the hourly execution profit $\pi_{i}$ of application $a_{i}$, so that, each application $a_{i}$ with higher hourly execution profit $\pi_{i}$ has higher priority from the user's view. Table 2 . shows the three applications that we consider in our running example and their related parameters. As can be seen, applications streamApp, enterpriseApp, have the priority of first (high), second (medium), and third (low) priority from the user's view, respectively.

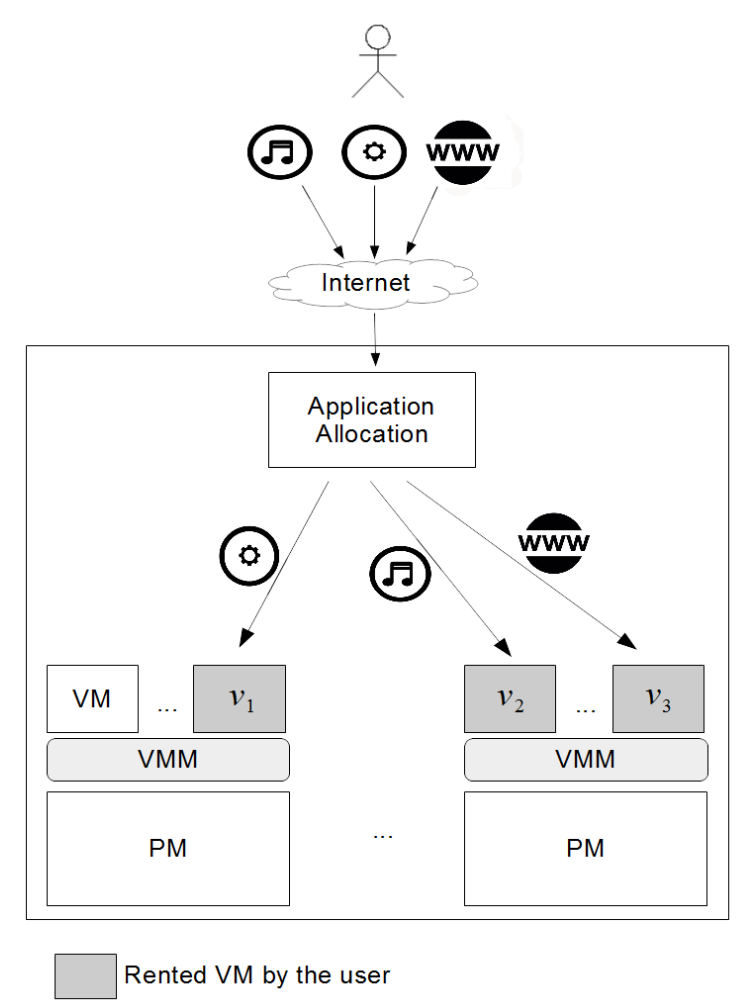

Fig.3. Reference Scenario

The user needs to assign each application in $A$ to a VM in a set $V=\left\{v_{1}, \ldots, v_{n}\right\}$ of rented VMs by her, which are running on multiple physical machines (PM). Each $v_{j} \hat{I} V$ is then associated with a characteristic double $V C_{j}=\left(c_{j}, L_{j}\right)$, where $c_{j}$ is the rental hourly charge of $v_{j}$ in US dollar terms. $L_{j}[1, \ldots, d]$ is the HUP level vector for $v_{j}$, where $L_{j}[k]$ represents the $k-t$ th HUP level of VM $v_{j}$. We assume that, in each hour, HUP of each $\mathrm{VM} v_{j} \in V$ falls in one of HUP levels defined in $L_{j}$. Also, Lev ${ }^{(j)}=\left\{\operatorname{Lev}_{1}^{(j)}, \ldots\right.$, Lev $\left._{d}^{(j)}\right\}$ denotes a set of characteristic quadruples, where quadruple Lev ${ }_{k}^{(j)}=\left(\min _{k}, \max _{k}, \operatorname{Pr}_{k}^{(j)}, \Gamma_{k}\right)$ is as sociated with the $H U P$ level $L_{j}[k] \cdot \min _{k}$ and $\max _{k}$ are the minimum and maximum values (in percent) for $H U P$ level $L_{j}[k]$, respectively.

Table 2. The user's applications

\begin{tabular}{|c|c|c|c|}
\hline $\begin{array}{c}\text { Application } \\
\text { Name }\end{array}$ & Service Type & $\begin{array}{c}\text { Hourly Profit } \\
(\$)\end{array}$ & Priority \\
\hline streamApp & Music Streaming & 4.9 & 1 \\
\hline enterpriseApp & $\begin{array}{c}\text { Complex Business } \\
\text { Logic }\end{array}$ & 4.5 & 2 \\
\hline webApp & LowTraffic Website & 2.1 & 3 \\
\hline
\end{tabular}


Table 3. Rented VMs by the user and their related parameters

\begin{tabular}{|c|c|c|c|c|c|c|}
\hline VM Name & $\begin{array}{l}\text { Rental Hourly } \\
\text { Charge } \\
\text { (c) }\end{array}$ & HUP Level & $\min H U P(\%)$ & $\max H U P(\%)$ & $\begin{array}{l}\text { Probability of } \\
\text { Occurrence } \\
\text { (Pr) }\end{array}$ & $\begin{array}{l}\text { Service Credit } \\
(\Gamma)\end{array}$ \\
\hline \multirow{4}{*}{$v_{1}$} & \multirow{4}{*}{0.015} & 1 & 99.96 & 100 & 0.997 & 0 \\
\hline & & 2 & 99 & 99.95 & 0.002 & 30 \\
\hline & & 3 & 95 & 98.99 & 0.0009 & 50 \\
\hline & & 4 & 90 & 94.99 & 0.0001 & 60 \\
\hline \multirow{4}{*}{$v_{2}$} & \multirow{4}{*}{0.004} & 1 & 99.96 & 100 & 0.96 & 0 \\
\hline & & 2 & 99 & 99.95 & 0.0395 & 30 \\
\hline & & 3 & 95 & 98.99 & 0.0004 & 50 \\
\hline & & 4 & 90 & 94.99 & 0.0001 & 60 \\
\hline \multirow{4}{*}{$v_{3}$} & \multirow{4}{*}{0.001} & 1 & 99.96 & 100 & 0.86 & 0 \\
\hline & & 2 & 99 & 99.95 & 0.03 & 30 \\
\hline & & 3 & 95 & 98.99 & 0.095 & 50 \\
\hline & & 4 & 90 & 94.99 & 0.015 & 60 \\
\hline
\end{tabular}

$(j)$

$\operatorname{Pr}_{k}$ and $\Gamma_{k}$ are the probability that $H U P$ of $\mathrm{VM} v_{j}$ falls in the HUP level $L_{j}[k]$ and the service credit of HUP level $L_{j}[k]$, respectively. Table 3 . shows the set of VMs in our running example. As can be seen there are three VMs, each with a rental hourly charge $\left(c_{j}\right)$. Also, each VM has four HUP levels $L_{j}[k]$, each with a proba(j)

bility of occurrence $\operatorname{Pr}_{k}$ and a service credit $\Gamma_{k}$. Referring to Table 3., for example, $V C_{1}=\left(0.015, L_{1}[1, \ldots, 4]\right)$ and Lev $_{3}^{(1)}=(95,98.99,0.0009,50)$.

Given a set of applications $A$ and a set of VMs $V$, a characteristic double $V C_{j}=\left(c_{j}, L_{j}\right)$ and a set of characteristic quadruples Lev ${ }^{(j)}=\left\{\right.$ Lev $_{1}{ }^{(j)}, \ldots$, Lev $\left._{d}{ }^{(j)}\right\}$, for each $v_{j} \in V$, a simple solution would select a cheaper VM for an application with higher priority. However, such a trivial approach may ignore the different availability levels of VMs, each with a different service credit and a probability of occurrence. Therefore, this approach might then be considered not desirable. To prevent such a situation, we propose to adopt a risk-aware approach aimed at choosing a VM $v_{j} \in V$ for an application $a_{i} \in A$, which maximizes the expected hourly pay-off $\pi_{i}$ of application $a_{i}$, considering the priority of applications. We consider our solution, as we will see in the next section, as a oneto-one allocation function alloc: $A \rightarrow V$, that takes the set $A$ of application instances as input and assigns each application $a_{i} \in A$ to a $\mathrm{VM} v_{j} \in V$ as output, so that, application $a_{i}$ has the highest expected hourly pay-off, if it is assigned to $v_{j}$, i.e., alloc $\left(a_{i}\right)=v_{j}$. Therefore, $v_{j}$ is an optimal VM for application $a_{i}$. Table 4. shows the notations that we will use in this paper together with their related descriptions.

Table 4. Notations

\begin{tabular}{|c|c|}
\hline Notation & Description \\
\hline$\pi_{i}$ & $\begin{array}{l}\text { average hourly financial execu- } \\
\text { tion profit of } a_{i}\end{array}$ \\
\hline$c_{j}$ & rental hourly charge of $v_{j}$ \\
\hline $\min _{k}$ & $\begin{array}{l}\text { minimum value of } H U P \text { of a VM } \\
\text { in level } k\end{array}$ \\
\hline $\max _{k}$ & $\begin{array}{l}\text { maximum value of } H U P \text { of a VM } \\
\text { in level } k\end{array}$ \\
\hline $\operatorname{Pr}_{k}^{(j)}$ & $\begin{array}{l}\text { probability that } H U P \text { of } v_{j} \text { falls } \\
\text { in level } l_{k}\end{array}$ \\
\hline$\Gamma_{k}$ & $\begin{array}{l}\text { service credit of } H U P \text { level } l_{k} \\
\text { for each VM }\end{array}$ \\
\hline$\xi_{j}[k]$ & $\begin{array}{l}\text { hourly penalty of } H U P \text { level } l_{k} \\
\text { for } v_{j}\end{array}$ \\
\hline$b_{j}[k]$ & $\begin{array}{l}\text { expected hourly benefit of cur- } \\
\text { rent application } a_{i} \text { in } H U P \text { level } \\
l_{k} \text {, if } \operatorname{alloc}\left(a_{i}\right)=v_{j}\end{array}$ \\
\hline$\theta_{i}[j]$ & $\begin{array}{l}\text { expected hourly pay-off for } a_{i} \text {, } \\
\text { if } \operatorname{alloc}\left(a_{i}\right)=v_{j}\end{array}$ \\
\hline
\end{tabular}

\section{PROPOSED APPROACH}

Our approach to choose a VM in $V$ for each application in $A$, so that, the expected hourly pay-off of each application is maximized, considering the priority of applications, operates in five steps (See Fig.5.): 1) Sorting the set $A ; 2)$ hourly penalty calculation of for each VM in $V ; 3)$ expected hourly benefit of each application if it is assigned to each VM in $V$; 4) expected hourly pay-off calculation of each application if it is assigned to each $\mathrm{VM}$ in $\mathrm{V}$; 5) update the set $V$. In the following, we present out approach in detail. 


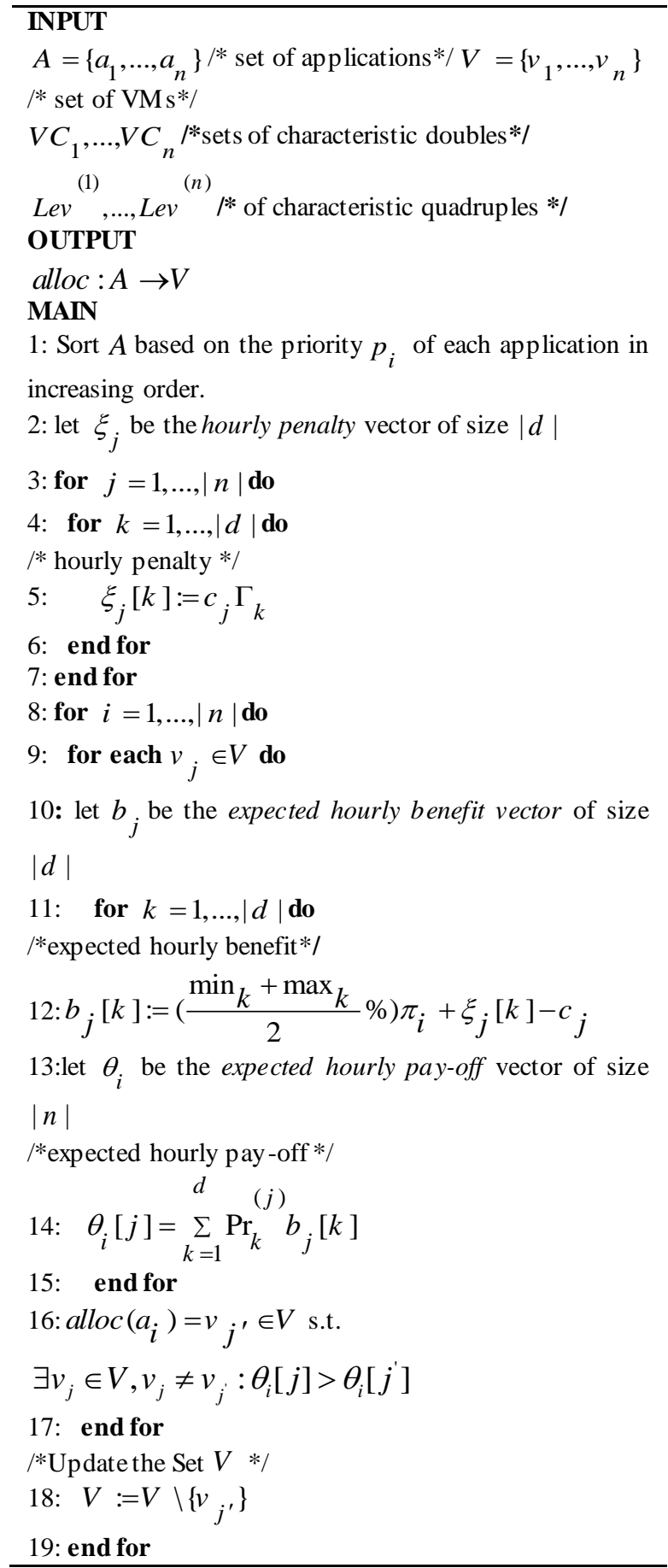

Fig.5. Algorithm for risk-aware assignment of the applications to the $\mathrm{VMs}$

Sorting. As we discussed earlier, in Section 0, in our proposed solution, the application-to-VM assignment is based on the priority of applications, defined by the user.

Therefore, first, we sort the set $A$ based on the priority of application $a_{i}$ in increasing order (line 1). Therefore, more important applications will be assigned to VMs that are more suitable for them in order to maximize their expected hourly pay-off. Therefore, referring to our running example, if we sort the set $A$ based on the priority of applications in increasing order, $A=\{$ streamApp,enterpriseApp,webApp $\}$.
Hourly penalty. Next, we calculate, for each VM $v_{j} \in V$, the hourly penalty, in each HUP level $L_{j}[k]$. To do this, we build a vector of hourly penalty $\xi_{j}[1, \ldots, d]$, where $\xi_{j}[k]=c_{j} \Gamma_{k}$, is the hourly penalty of $\mathrm{VM} v_{j}$ in HUP level $L_{j}[k]$ (lines 2-7). Back to our running example, Table 5. shows the calculation of hourly penalty $\xi$ of each $\mathrm{VM} v_{j} \in V$, in each $H U P$ level $l_{k}$. For instance, the hourly penalty of $\mathrm{VM} v_{1}$ in $H U P$ level $L_{1}[2]$ is $\xi_{1}[2]=c_{1} \Gamma_{2}=0.30 * 0.015=\$ 0.0045$.

In the remainder of this section, we illustrate in detail, our proposed solution. For simplicity, in the following, we refer our discussion to one application only (streamApp), with the note that the process described is executed for all applications in $A$.

Expected hourly benefit. For each $v_{j} \in V$, we compute the expected hourly benefit of application $a_{i}$ in $H U P$ level $l_{k}$ (lines 8-12). To do this, we build a vector of expected hourly benefit $b_{j}[1, \ldots, d]$, where $b_{j}[k]$ is the expected hourly benefit of application $a_{i}$, in $H U P$ level $L_{j}[k]$ of $v_{j}$, if $\operatorname{alloc}\left(a_{i}\right)=v_{j}$. It is given by:

$$
b_{j}[k]:=\left(\frac{\min _{k}+\max _{k}}{2} \%\right) \pi_{i}+\xi_{j}[k]-c_{j}
$$

Table 6. depicts the calculation of expected hourly benefit $b_{j}[k]$ of application streamApp for each $v_{j} \in V$, in each $H U P$ level $L_{j}[k]$. For example, the expected hourly benefit $b_{1}[2]$ of application streamApp in the second HUP level $L_{1}[2]$ of $v_{1}$ is equal to $b_{1}[2]=\left(\frac{99+99.95}{2} \%\right) 4.9+0.0045-0.0015=\$ 4.863$. Note that, while other approaches such as mean, median, etc. could be used, we consider the midrange of each HUP level $L_{j}[k]$, using $\min _{k}$ and $\max _{k}$, to calculate $b_{j}[k]$.

Expected hourly pay-off. In order to consider the uncertainty in assigning the current application (streamApp in our running example) to a $\mathrm{VM} v_{j} \in V$, so that, the expected hourly pay-off of current application, that is, streamApp in our running example, is maximized, we then propose to adopt a risk analys is technique, that permits to take into account of the probability of occurrence $\operatorname{Pr}_{k}^{(j)}$ of each HUP level $L_{j}[k]$ of each VMv $v_{j} \in V$. 
Table 5. Calculation of hourly penalty for each VM

\begin{tabular}{|c|c|c|c|c|}
\hline VM Name & $\begin{array}{l}\text { Rental Hourly Charge } \\
\text { (c) }\end{array}$ & HUP Level & $\begin{array}{c}\text { Service Credit } \\
(\Gamma)\end{array}$ & $\begin{array}{c}\text { Hourly Penalty } \\
\qquad(\xi)\end{array}$ \\
\hline \multirow{4}{*}{$v_{1}$} & \multirow{4}{*}{0.015} & 1 & $\overline{0}$ & 0 \\
\hline & & 2 & 30 & 0.0045 \\
\hline & & 3 & 50 & 0.0075 \\
\hline & & 4 & 60 & 0.009 \\
\hline \multirow{4}{*}{$v_{2}$} & \multirow{4}{*}{0.004} & 1 & 0 & 0 \\
\hline & & 2 & 30 & 0.0012 \\
\hline & & 3 & 50 & 0.002 \\
\hline & & 4 & 60 & 0.0024 \\
\hline \multirow{4}{*}{$v_{3}$} & \multirow{4}{*}{0.001} & 1 & 0 & 0 \\
\hline & & 2 & 30 & 0.0003 \\
\hline & & 3 & 50 & 0.0005 \\
\hline & & 4 & 60 & 0.0006 \\
\hline
\end{tabular}

Table 6. Expected hourly benefit for streamApp considering each VM

\begin{tabular}{|c|c|c|c|c|c|c|}
\hline $\begin{array}{l}\text { VM } \\
\text { Name }\end{array}$ & $\begin{array}{l}\text { Rental Hourly Charge } \\
\text { (c) }\end{array}$ & $\begin{array}{l}H U P \\
\text { Level }\end{array}$ & $\begin{array}{c}\min H U P \\
(\%)\end{array}$ & $\begin{array}{c}\max H U P \\
(\%)\end{array}$ & $\begin{array}{l}\text { Hourly penalty } \\
(\xi)\end{array}$ & $\begin{array}{l}\text { Expected Hourly Benefit } \\
\text { (b) }\end{array}$ \\
\hline \multirow{4}{*}{$v_{1}$} & \multirow{4}{*}{0.015} & 1 & 99.96 & 100 & 0 & 4.884 \\
\hline & & 2 & 99 & 99.95 & 0.0045 & 4.863 \\
\hline & & 3 & 95 & 98.99 & 0.0075 & 4.745 \\
\hline & & 4 & 90 & 94.99 & 0.009 & 4.526 \\
\hline \multirow{4}{*}{$v_{2}$} & \multirow{4}{*}{0.004} & 1 & 99.96 & 100 & 0 & 4.895 \\
\hline & & 2 & 99 & 99.95 & 0.0012 & 4.871 \\
\hline & & 3 & 95 & 98.99 & 0.002 & 4.750 \\
\hline & & 4 & 90 & 94.99 & 0.0024 & 4.530 \\
\hline \multirow{4}{*}{$v_{3}$} & \multirow{4}{*}{0.001} & 1 & 99.96 & 100 & 0 & 4.898 \\
\hline & & 2 & 99 & 99.95 & 0.0003 & 4.873 \\
\hline & & 3 & 95 & 98.99 & 0.0005 & 4.752 \\
\hline & & 4 & 90 & 94.99 & 0.0006 & 4.531 \\
\hline
\end{tabular}

While noting that there are different approaches can be applied (e.g., expected opportunity loss [9]), we consider the expected monetary value (EMV) [9]. Taking into account of the discussion in Section III.D, in our cloud scenario, events correspond to HUP levels of VMs. Therefore, we build a vector of expected hourly pay-off $\theta_{i}[1, \ldots, n]$ for application $a_{i}, \quad$ where $\theta_{i}[j]=\sum_{k=1}^{d} \operatorname{Pr}_{k}^{(j)} b_{j}[k]$, is the expected hourly pay-off of application $a_{i}$, if alloc $\left(a_{i}\right)=v_{j}$ (lines 13-14). Table 7 . shows the calculation of expected total hourly pay-off $\theta_{1}$ for the application streamApp, considering each VM in $V$.

For instance, the expected hourly pay-off of application streamApp, if it is assigned to $v_{2}$, is equal to

$\theta_{1}[2]=\sum_{1 \leq k \leq 4} \operatorname{Pr}_{k}^{(2)} b_{2}[k]=0.96 * 4.895+0.0395 * 4.871+0.0004 * 4.750$ $+0.0001 * 4.530=\$ 4.893$.

Therefore, $\theta_{1}=[4.883,4.893,4.877]$. We assign the current application $a_{i}$ to a VM, which it has the highest expected hourly pay-off with (line16).

Referring to our running example, the current application, streamApp, which is the most important application from the user's view, is assigned to $v_{2}$, i.e., alloc $($ streamApp $)=v_{2}$ because application streamApp has the highest expected hourly pay-off, if it is assigned to $v_{2}$, that is, $\theta_{1}[2]=\$ 4.893$. It is interesting to see that the chosen VM is not the cheapest VM in $V$. However, considering the different probability of occurrence and hourly penalty for each HUP level, the application streamApp is assigned to $v_{2}$, using EMV technique.

Updating. Finally, since we assume that only one application can be executed on a VM (alloc $: A \rightarrow V$ function is a one-to-one allocation function), after assigning application $a_{i}$ to $v_{j}$, we update the set of VMs $V$ by removing $v_{j}$ from $V$ (line 18). Therefore, considering our running example, $V=\left\{v_{1}, v_{3}\right\}$. 
Table 7. Expected hourly pay-off for streamApp considering each VM

\begin{tabular}{|c|c|c|c|c|}
\hline VM Name & HUP Level & $\begin{array}{l}\text { Probability of Occurrence } \\
\text { (Pr) }\end{array}$ & $\begin{array}{l}\text { Expected Hourly Benefit } \\
\text { (b) }\end{array}$ & $\begin{array}{l}\text { Expected hourly pay-off } \\
\qquad(\theta)\end{array}$ \\
\hline \multirow{4}{*}{$v_{1}$} & 1 & 0.997 & 4.884 & \multirow{4}{*}{4.883} \\
\hline & 2 & 0.002 & 4.863 & \\
\hline & 3 & 0.0009 & 4.745 & \\
\hline & 4 & 0.0001 & 4.526 & \\
\hline \multirow{4}{*}{$v_{2}$} & 1 & 0.96 & 4.895 & \multirow{4}{*}{4.893} \\
\hline & 2 & 0.0395 & 4.871 & \\
\hline & 3 & 0.0004 & 4.750 & \\
\hline & 4 & 0.0001 & 4.530 & \\
\hline \multirow{4}{*}{$v_{3}$} & 1 & 0.86 & 4.898 & \multirow{4}{*}{4.877} \\
\hline & 2 & 0.03 & 4.873 & \\
\hline & 3 & 0.095 & 4.752 & \\
\hline & 4 & 0.015 & 4.531 & \\
\hline
\end{tabular}

Fig.4, shows a decision analysis tree for the selection of a VM for application streamApp with maximum expected hourly pay-off. As can be seen, the decision tree is composed of three nodes that represent the VMs in $V$. For each VM, there are four leaf nodes, which each of them illustrates the expected hourly benefit $b_{j}[k]$ of
streamApp for $H U P$ level $L_{j}[k]$. If we continue to assign applications in $A$ to $\mathrm{VMs}$ in $V$, according to our proposed approach, alloc(enterpriseApp $)=v_{1} \quad$ and alloc $(w e b A p p)=v_{3}$.

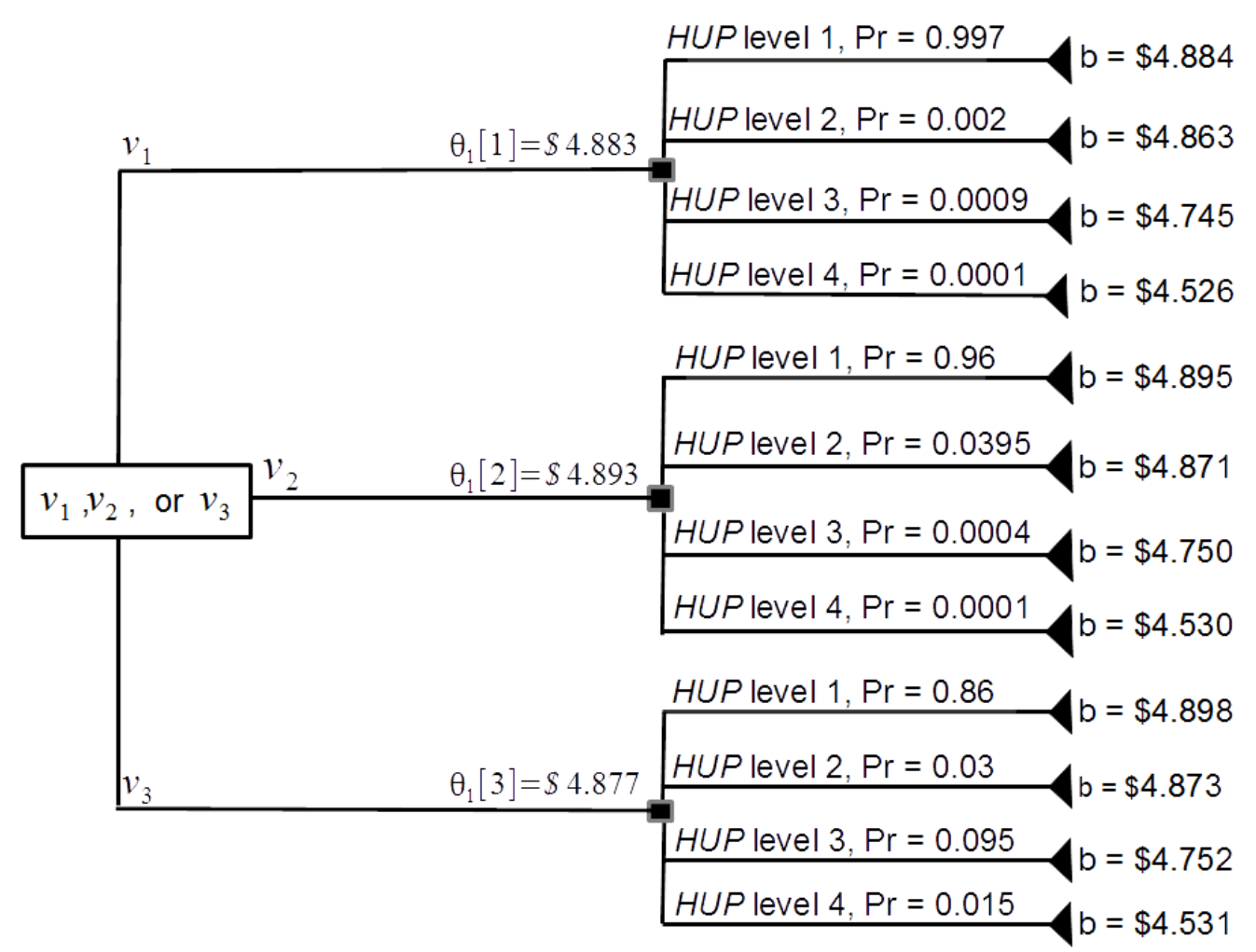

Fig.4. Decision tree to find an optimal VM for StreamApp

\section{CONCLUSIONS AND FUTURE WORK}

The paper presented a model for financial risk-aware application scheduling in cloud computing scenarios using risk analysis. We were interested in assigning the applications to the VMs, so that, the expected total pay- off for each application is maximized, considering the priority of applications, defined by the user. The proposed solution satisfies this requirement by considering several availability levels for a VM, each with a probability of occurrence and a service credit as a penalty rate.

In the proposed approach, we did not consider the risk attitude of a user [41] in assigning the applications to the 
VMs, including 1) risk aversion, e.g., if the applications of user are real time applications that cannot tolerate unavailability; 2) risk seeking, e.g., if the applications of user are time invariant, like offline down loading, which can be performed even with risk loss during the transaction; 3) risk neutral, e.g., if the applications of user can tolerate the risk of delay for a certain time period. As a future work, we will focus on the estimation of risk in application-to-VM assignment in cloud computing scenarios, using risk analysis by segregating users into different categories based on their risk behavior.

\section{REFERENCES}

[1] M. Y. Saeed and M. N. A. Khan, "Data Protection Techniques for Building Trust in Cloud Computing," Int. J. Mod. Educ. Comput. Sci., vol. 7, no. 8, p. 38, 2015 , "doi:10.5815/ijmecs.2015.08.05".

[2] A. Arman, A. Al-Shishtawy, and V. Vlassov, "Elasticity Controller for Cloud-Based Key-Value Stores.," Parallel Distrib. Syst. Int. Conf., pp. 268-275, 2012, "doi:10.1109/ICPADS.2012.45".

[3] A. Zia and M. N. A. Khan, "A Scheme to Reduce Response Time in Cloud Computing Environment," Int. J. Mod. Educ. Comput. Sci., vol. 5, no. 6, p. 56, 2013, "doi:10.5815/ijmecs.2013.06".

[4] S. Rajan and A. Jairath, "Cloud computing: The Fifth Generation of Computing," in Communication Systems and Network Technologies (CSNT), 2011 International Conference on, 2011, pp. 665-667, "doi: 10.1109/CSNT.2011.143".

[5] P. Mell and T. Grance, "The NIST Definition of Cloud Computing," 2011, "doi: 10.6028/NIST.SP.800-145".

[6] S. Lee and K.-K. Seo, "A Hybrid Multi-criteria Decisionmaking Model for a Cloud Service Selection Problem Using BSC, Fuzzy Delphi Method and Fuzzy AHP," Wirel. Pers. Commun., vol. 86, no. 1, pp. 57-75, 2016, "doi: 10.1007/s11277-015-2976-z".

[7] "Amazon Elastic Compute Cloud (Amazon EC2)." [Online]. Available: http://aws.amazon.com/ec2/. [Accessed: 11-Feb-2016].

[8] "Amazon EC2 Service Level Agreement." [Online]. Available:https://aws.amazon.com/ec2/sla/. [Accessed: 11-Feb-2016].

[9] D. M. Levine, M. L. Berenson, D. Stephan, and others, Statistics for managers using Microsoft Excel, vol. 660. Prentice Hall Upper Saddle River, NJ, 1999.

[10] X. Tang, K. Li, M. Qiu, and E. H.-M. Sha, "A Hierarchical Reliability-Driven Scheduling Algorithm in Grid Systems," J. Parallel Distrib. Comput., vol. 72, no. 4 pp. 525-535, 2012, "doi: 10.1016/j.jpdc.2011.12.004".

[11] H. N. Van, F. D. Tran, and J.-M. Menaud, "Performance and Power Management for Cloud Infrastructures," in Cloud Computing (CLOUD), 2010 IEEE 3rd International Conference on, 2010, pp. 329-336, "doi: 10.1109/CLOUD.2010.25".

[12] S. Zhang, B. Wang, B. Zhao, and J. Tao, “An EnergyAware Task Scheduling Algorithm for a Heterogeneous Data Center," in 2013 12th IEEE International Conference on Trust, Security and Privacy in Computing and Communications, 2013, pp. 1471-1477, "doi: 10.1109/TrustCom.2013.178".

[13] M. Sun, T. Zang, X. Xu, and R. Wang, "ConsumerCentered Cloud Services Selection Using AHP," in 2013 International Conference on Service Sciences (ICSS), 2013, pp. 1-6, "doi: 10.1109/ICSS.2013.26 ".
[14] V. C. Emeakaroha, I. Brandic, M. Maurer, and I. Breskovic, "SLA-Aware Application Deployment and Resource Allocation in Clouds," in Computer Software and Applications Conference Workshops (COMPSACW), 2011 IEEE 35th Annual, 2011, pp. 298-303, "doi: 10.1109/COMPSACW.2011.97".

[15] K. Black, Business statistics: for contemporary decision making. John Wiley \& Sons, 2011.

[16] S. K. Garg, S. K. Gopalaiy engar, and R. Buyya, "SLABased Resource Provisioning for Heterogeneous Workloads in a Virtualized Cloud Datacenter," in Algorithms and Architectures for Parallel Processing, Springer, 2011, pp. 371-384, "doi: 10.1007/978-3-64224650-0_32".

[17] C. S. Pawar and R. B. Wagh, "Priority based dynamic resource allocation in Cloud computing with modified waiting queue," in Intelligent Systems and Signal Processing (ISSP), 2013 International Conference on, 2013, pp. 311-316, "doi: 10.1109/ISSP.2013.6526925 ".

[18] D. C. Devi and V. R. Uthariaraj, "Load Balancing in Cloud Computing Environment Using Improved Weighted Round Robin Algorithm for Nonpreemptive Dependent Tasks," Sci. World J., vol. 2016, 2016, "doi: 10.1155/2016/3896065".

[19] R. Buyya, S. Pandey, and C. Vecchiola, "Cloudbus Toolkit for Market-oriented Cloud Computing," in Cloud Computing, Springer, 2009, pp. 24-44, "doi: 10.1007/9783-642-10665-1_4".

[20] L. Wang, G. Von Laszewski, A. Younge, X. He, M. Kunze, J. Tao, and C. Fu, "Cloud computing: A Perspective Study," New Gener. Comput., vol. 28, no. 2, pp. 137-146, 2010, "doi: 10.1007/s00354-008-0081-5".

[21] "VMware Infrastructure as a Service." [Online]. Available:https://www.vmware.com/support/services/iaasproduction. [Accessed: 25-May-2016].

[22] "Google App Engine Documentation." [Online]. Available:https://www.vmware.com/support/services/iaasproduction. [Accessed: 25-May-2016].

[23] "Amazon SimpleDB." [Online]. Available: https://aws.amazon.com/simpledb/. [Accessed: 25-May2016].

[24] "Salesforce.com." [Online]. Available: http://salesforce.com/. [Accessed: 25-May-2016].

[25] "Rackspace." [Online]. Available: https://www.rackspace.com/cloud. [Accessed: 25-May2016].

[26] P. Patel, A. H. Ranabahu, and A. P. Sheth, "Service level agreement in cloud computing," 2009.

[27] Y. Wang, S. Chen, and M. Pedram, "Service Level Agreement-Based Joint Application Environment Assignment and Resource Allocation in Cloud Computing Systems," in Green Technologies Conference, 2013 IEEE, 2013, pp. 167-174, "doi: 10.1109/GreenTech.2013.33".

[28] K. Bernsmed, M. G. Jaatun, P. H. Meland, and A. Undheim, "Security SLAs for Federated Cloud Services," in Availability, Reliability and Security (ARES), 2011 Sixth International Conference on, 2011, pp. 202-209, "doi: 10.1109/ARES.2011.34".

[29] A. V. Dastjerdi, S. G. H. Tabatabaei, and R. Buyya, “A dependency-aware Ontology-based Approach for Deploying Service Level Agreement Monitoring Services in Cloud," Softw. Pract. Exp., vol. 42, no. 4, pp. 501-518, 2012, "doi: 10.1002/spe.1104".

[30] P. Barham, B. Dragovic, K. Fraser, S. Hand, T. Harris, A. Ho, R. Neugebauer, I. Pratt, and A. Warfield, "Xen and the Art of Virtualization," ACM SIGOPS Oper. Syst. Rev., vol. 37, no. 5, pp. 164-177, 2003, "doi: 
10.1145/945445.945462".

[31] R. Buyya, C. S. Yeo, S. Venugopal, J. Broberg, and I. Brandic, "Cloud Computing and Emerging IT Platforms: Vision, Hype, and Reality for Delivering Computing as the 5th Utility," Futur. Gener. Comput. Syst., vol. 25, no. 6 pp. 599-616, 2009, 2009, "doi: 10.1016/j.future.2008.12.001".

[32] R. Jain and S. Paul, "Network Virtualization and Software Defined Networking for Cloud Computing: a Survey," IEEE Commun. Mag., vol. 51, no. 11, pp. 24-31, 2013, "doi:10.1109/MCOM.2013.6658648".

[33] M. Shiraz, S. Abolfazli, Z. Sanaei, and A. Gani, “A Study on Virtual Machine Deployment for Application Outsourcing in Mobile Cloud Computing," $J$. Supercomput., vol. 63, no. 3, pp. 946-964, 2013, "doi: 10.1007/s11227-012-0846-y".

[34] F. Zhang, J. Cao, K. Hwang, and C. Wu, "Ordinal Optimized Scheduling of Scientific Workflows in Elastic Compute Clouds," in Cloud Computing Technology and Science (CloudCom), 2011 IEEE Third International Conference on, 2011, pp.9-17.

[35] F. Lao, X. Zhang, and Z. Guo, "Parallelizing Video Transcoding Using Map-reduce-Based Cloud Computing," in Circuits and Systems (ISCAS), 2012 IEEE International Symposium on, 2012, pp. 2905-2908, "doi: 10.1109/ISCAS.2012.6271923".

[36] T. Xie, X. Qin, and A. Sung, "SAREC: A Security-Aware Scheduling Strategy for Real-time Applications on Clusters," in Parallel Processing, 2005. ICPP 2005. International Conference on, 2005, pp. 5-12, "doi: 10.1109/ICPP.2005.68".

[37] M. A. Arfeen, K. Pawlikowski, and A. Willig, "A Framework for Resource Allocation Strategies in Cloud Computing Environment," in Computer Software and Applications Conference Workshops (COMPSACW), 2011 IEEE 35th Annual, 2011, pp. 261-266, "doi: 10.1109/COMPSACW.2011.52".

[38] F. López, M. A. Amutio, J. Candau, and J. A. Mañas, "Methodology for Information Systems Risk Analy sis and Management," Minist. Public Adm., 2005.

[39] T. Aven, "Risk analysis. Assessing uncertaintie beyond expected values and probabilities, 2008." Wiley, Chichester, UKT, "doi: 10.1002/9780470694435".

[40] M. J. Thaheem, A. De Marco, and K. Barlish, "A Review of Quantitative Analysis Techniques for Construction Project Risk Management," in Proceedings of the Creative Construct Conference, 2012, pp. 656-667.

[41] K. Gokulnath and R. Uthariaraj, "Game Theory Based Trust Model for Cloud Environment," Sci. World J., vol. 2015, 2015, "doi: 10.1155/2015/709827".

\section{Authors' Profiles}

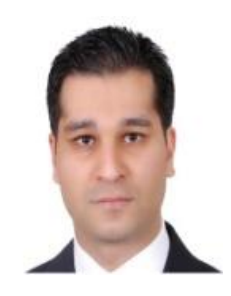

Ala Arman was born on September 4, 1982. He received his master's degree in "software engineering of distributed systems" at Royal Institute of Technology (KTH), Stockholm, Sweden, in 2012. His master's dissertation was on "Automated Control of Elasticity for a Cloud-Based Key-Value Store". In 2014, he joined as a $\mathrm{PhD}$ researcher at the computer science department of the Università degli Studi di Milano. His research interests include security and resource management in cloud computing.
How to cite this paper: Ala Arman, "A Risk-Aware Application Scheduling Model in Cloud Computing Scenarios, International Journal of Intelligent Sy stems and Applications (IJISA), Vol.8, No.10, pp.11-20, 2016. DOI: 10.5815/ijisa.2016.10.02 